\title{
ON TOPOLOGICAL METHODS IN HOMOLOGICAL ALGEBRA
}

\author{
DAVID A. EDWARDS AND HAROLD M. HASTINGS ${ }^{1}$
}

\begin{abstract}
We give an appropriate extension of the concept of "tower of surjections" to arbitrary inverse systems. We introduce a natural closed model structure (in the sense of D. Quillen) on the category of pro-(Simplicial Abelian Groups) and interpret our condition as the definition of fibrant object.
\end{abstract}

1. Introduction. Consider the following well-known proposition.

(1.1) Proposition. Let

$$
G_{0} \leftarrow \cdots \leftarrow G_{i} \leftarrow G_{i+1} \leftarrow \ldots
$$

be a tower of surjections of abelian groups. Then

(a) the natural maps $\lim \left\{G_{i}\right\} \rightarrow G_{i_{0}}$ are surjections, and

(b) $\lim ^{s}\left\{G_{i}\right\}=0$ for $s>0$.

It is also well known (see, for example, J. Keesling [11]) that (a) fails in general for uncountable inverse systems bonded by surjections. We shall show in $\$ 2$ that (b) also fails in general for such systems. In $\$ 3$ we shall show that (a) and (b) both hold for inverse systems $\left\{G_{i}\right\}$ indexed by cofinite strongly directed sets with the property

$$
\text { the induced maps } G_{i_{0}} \rightarrow \lim _{i<i_{0}}\left\{G_{i}\right\} \text { are surjections. }
$$

A directed set $I=\{i\}$ called strongly directed if $i \leqslant i^{\prime}$ and $i^{\prime} \leqslant i$ imply $i=i^{\prime} . I$ is called cofinite if each element $i \in I$ has finitely many predecessors. In [6], [7], we extend a result of $S$. Mardešić [12] to show that every filtering category $J$ (a notion more general than directed set) is equivalent to a cofinite strongly directed set.

In $\S 4$ we shall interpret condition (1.2) as the definition of fibrant object in a suitable closed model structure (D. Quillen [14]) on pro-(Simplical Abelian Groups) [6], [7], [8], and indicate several applications.

Z. Z. Yeh [17], J.-E. Roos [15], and J.-L. Verdier [16] have shown that part (b) of Proposition (1.1) holds for flasque inverse systems; C. U. Jensen [10]

Presented to the Society, March 23, 1975; received by the editors February 4, 1975 and, in revised form, November 3, 1975 and December 22, 1975.

AMS (MOS) subject classifications (1970). Primary 55J99, 18G10; Secondary 18E10, 18E25, $55 \mathrm{D} 99$.

Key words and phrases. Pro-(abelian groups), pro-homotopy, limit, lims, closed model category.

${ }^{1}$ This research was begun while the second-named author held a visiting position at the State University of New York at Binghamton, whose support he gratefully acknowledges.

(1) American Mathematical Society 1976 
extended their results to weakly flasque systems. (A subset $J$ of a directed set $I$ is called ordered if $i \in J$ and $i^{\prime}<i$ imply $i^{\prime} \in J$. An inverse system $\left\{G_{i}\right\}$ of abelian groups indexed by a directed set $I$ is called flasque (resp., weakly flasque) if for each ordered (resp., directed) subset $J$ of $I$ the induced map $\lim _{i \in I}\left\{G_{i}\right\} \rightarrow \lim _{i \in J}\left\{G_{i}\right\}$ is surjective.) In $\$ 3$ we shall show that flasque is equivalent to condition (1.2).

(1.3) Convention. In the rest of this paper, we shall assume that all inverse systems are indexed by cofinite strongly directed sets.

A. Grothendieck [8] associated to a category $C$ the category pro- $C$ of inverse systems over $C$; see M. Artin and B. Mazur [1, Appendix] for a description of pro- $C$.

2. Inadequacy of the Mittag-Leffler condition. The inverse limit, lim, and its right derived functors $\lim ^{s}$ are functors from pro-(Abelian Groups) to Abelian Groups ([17], [4], see [6] for a proof).

(2.1) Definitions (see, e.g., [3]). (a) An inverse system $\left\{G_{i}\right\}$ is called movable if for each $i$ there exists a $j>i$ such that for any $k>j$ there exists a filler in the diagram

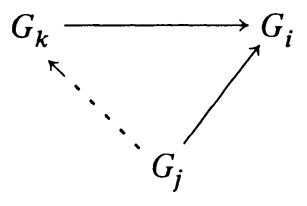

(b) $\left\{G_{i}\right\}$ is called Mittag-Leffler (M-L) if $\left\{G_{i}\right\}$ is isomorphic in pro-(Abelian Groups) to an inverse system $\left\{G_{j}^{\prime}\right\}$ whose bonding maps are surjections.

(2.2) Lemma (see, e.g., [3]). A movable inverse system is Mittag-Leffler.

We omit the routine proof.

(2.3) Proposition. There is a short exact sequence of Mittag-Leffler proabelian groups on which $\lim$ is not exact; hence $\lim ^{1}$ does not vanish on all Mittag-Leffler pro-abelian groups.

Proof. In [10], Keesling constructs a movable pair $\left\{\left(X_{i}, A_{i}\right)\right\}$ in the category pro-(ANR Pairs), hence a movable system of long exact sequences

$$
\left\{\ldots \rightarrow H_{1}\left(X_{i}\right) \rightarrow H_{1}\left(X_{i}, A_{i}\right) \stackrel{\partial_{i}}{\rightarrow} H_{0}\left(A_{1}\right) \rightarrow \ldots\right\}
$$

such that the sequence

$$
\lim \left\{H_{1}\left(X_{i}\right)\right\} \rightarrow \lim \left\{H_{1}\left(X_{i}, A_{i}\right)\right\} \rightarrow \lim \left\{H_{0}\left(A_{i}\right)\right\}
$$

is not exact at the middle term.

It is easy to show that the kernels and images in sequence (2.4) are movable, hence M-L. If lim were exact on such systems, we would have 


$$
\begin{gathered}
\operatorname{Im}\left(\lim \left\{H_{1}\left(X_{i}\right)\right\} \rightarrow \lim \left\{H_{1}\left(X_{i}, A_{i}\right)\right\}\right)=\lim \left\{\operatorname{Im}\left(H_{1}\left(X_{i}\right) \rightarrow H_{1}\left(A_{i}\right)\right)\right\} \\
=\operatorname{ker}\left(\lim \left\{H_{1}\left(X_{i}, A_{i}\right)\right\} \rightarrow \lim \left\{H_{0}\left(A_{i}\right)\right\}\right),
\end{gathered}
$$

in sequence (2.5), contradicting Keesling. Hence lim is not exact on M-L pro-abelian groups.

\section{The strong Mittag-Leffler condition.}

(3.1) Definition. An inverse system of abelian groups, $\left\{G_{i}\right\}$, indexed by a cofinite directed set, is said to satisfy the strong Mittag-Leffler (S-M-L) condition if, for each $i_{0}$, the induced map $G_{i_{0}} \rightarrow \lim _{i<i_{0}}\left\{G_{i}\right\}$ is surjective. More generally, any pro-abelian group isomorphic to an S-M-L pro-abelian group in pro-(Abelian Groups) will be called S-M-L.

(3.2) Remarks. Clearly S-M-L implies M-L, and the two conditions are equivalent on towers (inverse systems indexed by the natural numbers).

(3.3) Proposition. An inverse system $\left\{G_{i}\right\}$ is strongly Mittag-Leffler if and only if $\left\{G_{i}\right\}$ is isomorphic in pro-(Abelian Groups) to a flasque inverse system.

Proof. Clearly, if $\left\{G_{i}\right\}$ is flasque, the natural maps $G_{i_{0}} \rightarrow \lim _{i<i_{0}}\left\{G_{i}\right\}$ are surjections.

Conversely, assume that for each $i_{0}$ the natural maps $G_{i_{0}} \rightarrow \lim _{i<i_{0}}\left\{G_{i}\right\}$ are surjections. Let $I$ denote $\{i\}$, and let $J$ be an ordered subset of $I$. Given an element $g_{J}=\left\{g_{i} \mid i \in J\right\}$ of $G_{J}=\lim _{i \in J}\left\{G_{i}\right\}$ we shall find an element $g_{I}=$ $\left\{g_{i} \mid i \in I\right\}$ which extends $g_{J}$; this will show that $\left\{G_{i}\right\}$ is flasque, as required.

We shall define the required elements $g_{i}$ in $I \backslash J$ by induction. Suppose that for a given $i_{0}$, and for all $i<i_{0}$, elements $g_{i}$ in $G_{i}$ have been chosen so that for $i^{\prime}<i, g_{i}$ maps to $g_{i^{\prime}}$ in $G_{i^{\prime}}$ (If $i_{0}$ has no predecessors in $I \backslash J$, this assumption is clearly satisfied.) By hypothesis we may define $g_{i_{0}}$ in $G_{i_{0}}$ such that, for all $i<i_{0}, g_{i_{0}}$ maps to $g_{i}$ in $G_{i}$. Because $I$ is a cofinite strongly directed set, this process yields the required element $g_{I}$ in $\lim _{i \in I}\left\{G_{i}\right\}$.

4. Relation with pro-homotopy. We shall give a homotopy-theoretic interpretation of the S-M-L condition. D. Quillen [14, \$1.1] formalized axiomatic homotopy theory by introducing the concept of closed model category. A closed model category $C$ consists of an underlying category $C$ together with three classes of maps; cofibrations, fibrations, and equivalences. These maps are required to satisfy the "usual properties." In particular, given a solidarrow commutative diagram of the form

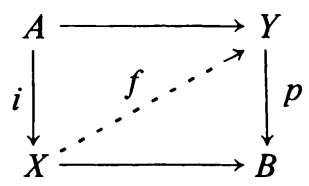

in which $i$ is a cofibration, $p$ is a fibration, and $i$ or $p$ is an equivalence, there 
exists a filler $f$. An object $X$ is called fibrant if the natural map $X \rightarrow *$ is a fibration.

The category $S S$ of simplicial sets (see, e.g., J. P. May [13]) is the prototype closed model category [14, §II.3]; the cofibrations are the inclusions, the fibrations are the Kan fibrations, and the equivalences are the weak homotopy equivalences. The category $S$ (Abelian Groups) of simplicial abelian groups inherits a closed model structure from $S S$ [14, §II.3]. Caution. Not all inclusions are cofibrations in $S$ (Abelian Groups). In the following discussion let $C$ be either $S S$ or $S$ (Abelian Groups). The case $C=S S$ was first developed in [9].

(4.2) Definitions [7]. A level map $\left\{f_{i}\right\}$ in pro- $C$ is a cofibration (resp., equivalence) if each $f_{i}$ is a cofibration (resp., equivalence). More generally, any composite of retracts of such maps is a cofibration (resp., equivalence). Fibrations are defined by lifting property (4.1).

(4.3) THEOREM [7]. pro- $C$ with the above structure is a closed model category. An object $X$ of pro- $C$ is fibrant if and only if $X$ is a retract of an object $\left\{Y_{i}\right\}$ indexed by a cofinite strongly directed set $\{i\}$ such that, for each $i_{0}$, the induced map $Y_{i_{0}} \rightarrow \lim _{i<i_{0}}\left\{Y_{i}\right\}$ is a fibration.

We relate Theorem (4.3) to the homological algebra of $\S 3$ as follows. Quillen [14, §II.3] showed that a map $f: G \rightarrow H$ of abelian groups is a surjection if and only if $f$ is a fibration in $S$ (Abelian Groups). Hence a pro-abelian group $X$ is S-M-L if and only if $X$ is fibrant in pro- $S$ (Abelian Groups). This allows us to give another proof of the following result of Yeh [17] and Roos [15]; see also Verdier [16] and Jensen [10].

(4.4) Theorem [6], [7] (compare Proposition (1.1)(b) above). For $s>0$, lims vanishes on $S-M-L$ pro-abelian groups.

\section{REFERENCES}

1. M. Artin and B. Mazur, Etale homotopy, Lecture Notes in Math., vol. 100, Springer-Verlag, Berlin and New York, 1969. MR 39 \#6883.

2. A. K. Bousfield and D. M. Kan, Homotopy limits, completions, and localizations, Lecture Notes in Math., vol. 304, Springer-Verlag, Berlin, 1972.

3. D. A. Edwards, Etale homotopy theory and shape, Lecture Notes in Math., vol. 428, Springer-Verlag, Berlin and New York, 1972, pp. 58-107.

4. D. A. Edwards and R. Geoghegan, Stability theorems in shape and pro-homotopy, Trans. Amer. Math. Soc. 222 (1976), 389-403.

5. D. A. Edwards and H. M. Hastings, Why the R-completion works, General Topology and Appl. (to appear).

6. On homotopy inverse limits and the vanishing of $\lim ^{s}$, S.U.N.Y. at Binghamton (preprint).

7. , Cech and Steenrod homotopy theory, with applications to geometric topology, Lecture Notes in Math., Springer-Verlag, Berlin and New York (to appear).

8. A. Grothendieck, Technique de descente et théorèmes d'existence en géométrie algébrique, Séminaire Bourbaki, 1959-1960, Exposés 190, 195 (mimeographed notes).

9. H. M. Hastings, Homotopy theory of pro-spaces. I, II, S.U.N.Y. at Binghamton, 1974 (preprint). 
10. C. U. Jensen, Les foncteurs dérivés de lim proj. et leurs applications en théorie des modules, Lecture Notes in Math., vol. 254, Springer-Verlag, Berlin and New York, 1972.

11. J. Keesling, The $\grave{C}$ ech homology of compact connected abelian topological groups with applications to shape theory, Lecture Notes in Math., vol. 438, Springer-Verlag, Berlin and New York (to appear).

12. S. Mardešic, On the Whitehead theorem in shape theory, Fund. Math. (to appear).

13. J. P. May, Simplicial objects in algebraic topology, Van Nostrand, Princeton, N. J., 1967. MR 36 \# 5942.

14. D. G. Quillen, Homotopical algebra, Lecture Notes in Math., vol. 43, Springer-Verlag, Berlin and New York, 1967. MR 36 \#6480.

15. J.-E. Roos, Sur les foncteurs dérivés de lim. Applications, C. R. Acad. Sci. Paris 252 (1961), 3702-3704. MR 24 \# A1938.

16. J.-L. Verdier, Équivalence essentielle des systèmes projectifs, C. R. Acad. Sci. Paris 261 (1965), 4950-4953. MR 32 \#7623.

17. Z. Z. Yeh, Thesis, Princeton, N. J., 1959.

Department of Mathematical Sciences, SUNY at Binghamton, Binghamton, New York 13901

Department of Mathematics, Hofstra University, Hempstead, New York 11550 